\title{
Sliding Mode Reference Coordination of Constrained Feedback Systems
}

\author{
Alejandro Vignoni, ${ }^{1}$ Fabricio Garelli, ${ }^{2}$ and Jesús Picó ${ }^{1}$ \\ ${ }^{1}$ Institut d'Automàtica i Informàtica Industrial, Universitat Politècnica de València, Camì de Vera s/n, 46022 València, Spain \\ ${ }^{2}$ CONICET and Facultad de Ingenieria, Universidad Nacional de La Plata (UNLP), Calle 48 esq. 116 s/n, 1900 La Plata, Argentina \\ Correspondence should be addressed to Alejandro Vignoni; alvig2@upv.es
}

Received 4 September 2013; Revised 20 November 2013; Accepted 21 November 2013

Academic Editor: Rongni Yang

Copyright (c) 2013 Alejandro Vignoni et al. This is an open access article distributed under the Creative Commons Attribution License, which permits unrestricted use, distribution, and reproduction in any medium, provided the original work is properly cited.

\begin{abstract}
This paper addresses the problem of coordinating dynamical systems with possibly different dynamics (e.g., linear and nonlinear, different orders, constraints, etc.) to achieve some desired collective behavior under the constraints and capabilities of each system. To this end, we develop a new methodology based on reference conditioning techniques using geometric set invariance and sliding mode control: the sliding mode reference coordination (SMRCoord). The main idea is to coordinate the systems references. Starting from a general framework, we propose two approaches: a local one through direct interactions between the different systems by sharing and conditioning their own references and a global centralized one, where a central node makes decisions using information coming from the systems references. In particular, in this work we focus in implementation on multivariable systems like unmanned aerial vehicles (UAVs) and robustness to external perturbations. To show the applicability of the approach, the problem of coordinating UAVs with input constraints is addressed as a particular case of multivariable reference coordination with both global and local configuration.
\end{abstract}

\section{Introduction}

Coordination of multiagents and, in particular, formation control of multiple unmanned aerial vehicles (UAVs) is a very up-to-date topic, and it supports many practical applications, such as surveillance, weather forecasting, damage assessment, and search and rescue [1-3].

Recently, the consensus problem was addressed using algebraic graph theory and properties of the Laplacian Matrix for single integrators (see $[1,4]$ and references therein). This approach was extended to a chain of integrators in [5], and to nonlinear multiagent systems in [6].

Sliding mode control is an important topic in nonlinear systems. Research in nonlinear systems goes from stability analysis (e.g., with fuzzy polynomial models and sum of squares tools [7]) to estimation (e.g., with second order sliding mode observers for kinetic rates [8]) to control (e.g., with bounded L2 gain performance of Markovian jump singular time-delay systems [9]).
The use of sliding mode (SM) techniques is generally used for control of swarms and multi-agent systems to achieve consensus. In those situations, a master-slave or leaderfollower configuration is implemented, and the discontinuous action is a control signal. In [10], higher-order sliding mode controllers are used in such configuration to maintain the formation shape. In [11], finite-time sliding mode estimators are used to achieve consensus in decentralized formation control with virtual leader. Also in [12], a discontinuous control input is chosen to be proportional to the gradient of a positive semidefinite disagreement function defined by the graph Laplacian matrix, leading to a sliding mode consensus algorithm. Recently in [13], consensus is achieved in connected and also in fully connected swarms of idealized and identical first-order dynamic systems enforced by sliding modes. Also in [14], finite-time consensus algorithms for a swarm of self-propelling agents based on sliding mode control and graph algebraic theories are developed. In [15], an LMI approach to multiagent systems control is performed 
under time delay and uncertainties. Finally, in [16], exact formation control is achieved with binary information of the position of the other agents.

In a recent proposal from one of the coauthors, sliding mode control has been used in a nontraditional way: the sliding mode reference conditioning (SMRC) technique [17]. This technique combines reference conditioning and sliding mode ideas and has been used in the beginning to bound cross-coupling interactions in multivariable linear systems $[18,19]$ and for set-point seeking in nonlinear systems with state dependent constraints [20]. In [21], an SMRC auxiliary loop has been implemented to reduce hypoglycemia in a closed-loop glucose control for DM type 1 patients. Also in [22], a geometric invariance and sliding mode ideas have been proposed for redundancy resolution in robotic systems. And in [23], an integrated solution based on the same ideas has been proposed for robotic trajectory tracking, path planning and speed autoregulation.

In the previous work [24-27], the authors use sliding mode reference conditioning to enforce coordination in multi-agent systems. In [24], sliding mode reference coordination in SISO systems is imposed with a global supervisory approach and two layers of SMRC in a hierarchical structure. In [25], the coordination arises from the local interaction between the systems, and the information flows in only one level, as the swarm is assumed to have no leader. In [26], the authors make a multivariable reference coordination for ideal unconstrained systems. A preliminary unifying work is done in [27] with focus on SISO systems. The present work is a unified approach of the sliding mode reference coordination (SMRCoord) integrating both local and global approaches with focus on implementation of multivariable systems and robustness of perturbation to the coordination goals.

The rest of the paper is organized as follows. Next section presents the problem of coordination in a general form. Then in Section 3, some previous results in set invariance and reference conditioning used thereafter to propose coordination strategy are described. In Section 4, a decentralized version is proposed to solve the coordination problem with only one hierarchical level and no leader. Meanwhile in Section 5, the supervised global coordination method is proposed. In Section 6, the proposed strategies are used to coordinate UAVs showing the result obtained by simulations. Finally, in Section 7, some conclusions are presented and open issues for future study are considered.

\section{Problem Statement}

In this section, a general form of the coordination problem is presented, together with definitions and assumptions regarding constrained systems and systems coordination.

Consider $N$ stable closed-loop systems with different dynamic behaviour, constraints, and performance. In the context of constrained systems, the following feasible reference concept arises [28]: the fastest reference a system can follow without violating its constraints while beiing in closed loop. In the case of a system with actuator saturation as a constraint, a feasible reference will never lead the actuators out of their operation range; otherwise it may open the loop, leaving the control system without feedback and leading to a windup effect.

Hereafter, coordination will be understood as the action needed in order to obtain a collective behaviour in a set of considered systems. In this work, the coordination problem is approached by acting on the systems references.

Among the possible collective behaviours, one can count

(i) keeping a function of the local references $(\chi)$ close to the global reference,

(ii) maintaining a fixed distance between the systems local references, one to one, or between flock centroids,

(iii) obtaining generalized synchronization, as a limit case of the previous cases.

Note the function $\chi$ may be some function of the local references, like the mean, mode, max, or min. As a consequence of this, the resulting definition of coordination is rather general and broad, depending on the selected $\chi$ function.

2.1. Information Exchange. Information exchange is one of the key elements when coordinating a set of systems. Depending on the level on which this information is exchanged as in the following architectures may arise Figure 1:

Local Topology when information flows in the same level among neighbours systems;

Global Topology when information exchange occurs in a higher hierarchical level, to a supervisory node.

Information exchange still is one of the main bottlenecks of a centralized topology: the data collection time depends on the number of systems involved unlike the decentralized one which depends only on the network radius. Moreover, the global topology presents the common issues of vulnerability and fragility. If the central node has a communication problem or if it is under attack, all the network gets compromised.

2.2. Constrained Systems. When working with constrained systems, if the constrained variables take different value from the desired ones, inadequate values of the state variables $x$ can destroy control performance. In order to restore the state adequacy, auxiliary inputs $\mathbf{r}_{f}(\mathbf{x})$, called feasible references, are used [28].

Definition 1. The feasible reference $\mathbf{r}_{f}(\mathbf{x})$ of a closed-loop system is the closest input to the original reference $r$ such that if $\mathbf{r}_{f}(\mathbf{x})$ had been applied to the controller instead of $r$, the system constraints would have not been violated; that is, the trajectories of the system and in particular the constrained variables would remain inside (or in the boundary of) a given set $\Phi$, defined by the system constraints.

\subsection{Assumptions and Definitions. Using the following assumption}

Assumption 2. Each system has a stabilizing control loop and can follow a feasible reference.

The coordination is defined as follows. 


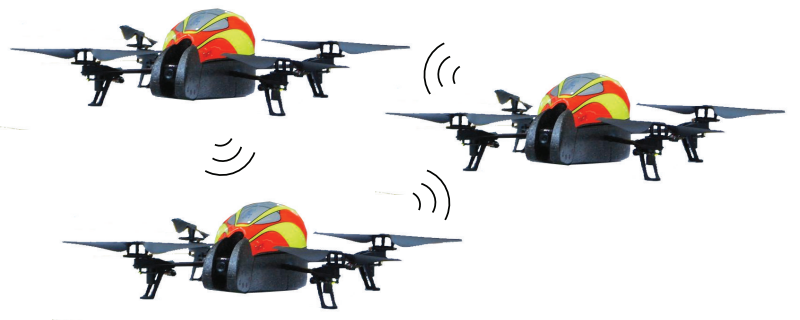

(a)

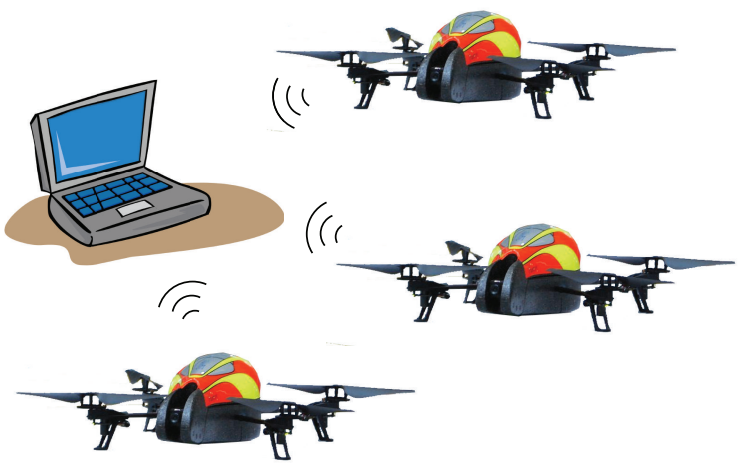

(b)

FIGURE 1: Network topology of the different architectures implemented in quadrotors. (a) Local distributed topology. (b) Supervised global topology.

Definition 3. The systems are said to be coordinated as long as their references belong to the invariant set $\Phi_{c}(\mathbf{x}, \rho)$, by modifying each system feasible reference. The set $\Phi_{c}(\mathbf{x}, \rho)$ is defined as

$$
\Phi_{c}(\mathbf{x}, \rho)=\left\{\mathbf{x} \in \mathbf{X}: \phi_{c}(\mathbf{x}, \rho)=\|\mathbf{r}(\mathbf{x})-\rho\|-\delta \leq 0\right\},
$$

where $\mathbf{x} \in \mathbf{X} \in \mathbb{R}^{n}$ are the system states, $\mathbf{r}(\mathbf{x})$ is a feasible reference function of the states $\mathbf{x}, \rho$ is a function depending on the information coming from the other systems, and $\delta$ is a predefined value. The norm $\|\cdot\|$ can be any norm defined in $\mathbb{R}^{n}$. However, hereafter we will use the Euclidean norm for simplicity.

\section{Geometric Set Invariance and Sliding Mode Reference Conditioning}

In this section, the methodology used in this paper to achieve the coordination of feasible references is described. It is based on concepts of geometric set invariance and sliding mode reference conditioning.

3.1. Geometric Set Invariance. Consider the following dynamical system:

$$
\Sigma:\left\{\begin{array}{l}
\dot{\mathbf{x}}=\mathbf{f}(\mathbf{x})+\mathbf{g}(\mathbf{x}) \mathbf{u} \\
\mathbf{y}=\mathbf{h}(\mathbf{x})
\end{array}\right.
$$

where $\mathbf{x} \in \mathbf{X} \subset \mathbb{R}^{n}$ is the state vector, $\mathbf{u} \in \mathbf{U} \subset \mathbb{R}^{m}$ is a control input (possibly discontinuous), $\mathbf{f}: \mathbb{R}^{n} \rightarrow \mathbb{R}^{n}$ and g : $\mathbb{R}^{n} \rightarrow \mathbb{R}^{n}$ are vector fields, and $\mathbf{h}: \mathbb{R}^{n} \rightarrow \mathbb{R}^{b}$ is scalar fields; all of them are defined in $\mathbf{X}$.

The variable $\mathbf{y}$ denotes the system output vector, which has to be bounded so as to fulfill $j=1, \ldots, N$ user-specified system constraints $\phi_{i}$. The corresponding bounds on $\mathbf{y}$ are given by the set:

$$
\Phi=\left\{\mathbf{x} \in \mathbf{X} \mid \phi_{i}(\mathbf{y}) \leq 0\right\}, \quad i=1, \ldots, N .
$$

From a geometrical point of view, the goal is to find a control input $\mathbf{u}$ such that the region $\Phi$ becomes invariant (i.e., trajectories originating in $\Phi$ remain in $\Phi$ for all times $t$ ), while $\mathbf{y}$ is driven as close as possible to its desired value $\mathbf{r}$.

To ensure the invariance of $\Phi$, the control input $\mathbf{u}$ must guarantee that the right hand side of the first equation in (2) points to the interior of $\Phi$ at all points on the boundary layer of $\Phi$, denoted by $\partial \Phi$, defined as

$$
\partial \Phi=\bigcup_{i=1}^{N} \partial \Phi_{i}, \quad \partial \Phi_{i}=\left\{\mathbf{x} \in \Phi \mid \phi_{i}(\mathbf{y})=0\right\} .
$$

The following assumption will be needed for later development and will allow us to compute the gradient vector $\nabla \phi_{j}$ of the functions $\phi_{i}$.

Assumption 4. All the $\phi_{i}$ functions are assumed to be differentiable in the boundary $\partial \Phi_{i}$.

Mathematically, the invariance of $\Phi$ may be ensured by an input $\mathbf{u}$, such that, for all $i, \dot{\phi}_{i} \leq 0$, when $\phi_{i}(\mathbf{y})=0$. This condition can be expressed as

$$
\begin{array}{r}
\dot{\phi}_{i}(\mathbf{x}, \mathbf{d}, \mathbf{u})=\nabla \phi_{i}^{\top} \dot{\mathbf{x}}=\left\|\nabla \phi_{i}\right\|\|\mathbf{f}+\mathbf{g u}\| \cos \theta \\
=\nabla \phi^{\top} \mathbf{f}+\nabla \phi^{\top} \mathbf{g u}=L_{f} \phi_{i}+\mathbf{L}_{\mathbf{g}} \phi_{i} \mathbf{u}, \\
\forall \mathbf{x} \in \partial \Phi_{i}, \quad j=1, \ldots, N,
\end{array}
$$

which constitute in standard form the implicit invariance condition $[29,30]$ :

$$
\inf _{\mathbf{u}}\left\{\dot{\phi}_{i}(\mathbf{x}, \mathbf{d}, \mathbf{u}) \leq 0, \forall \mathbf{x} \in \partial \Phi_{i}\right\}, \quad j=1, \ldots, N .
$$

Solving (6) for $\mathbf{u}$ results in the explicit invariance condition for system (2) and a particular constraint $\phi_{i}$. The set $\mathcal{U}_{i}$ of feasible solutions is obtained:

$$
\begin{aligned}
& \mathcal{U}_{i}(\mathbf{x}, \mathbf{d}) \\
& =\left\{\begin{array}{l}
\mathbf{u} \in\left\{\mathbf{U} \mid L_{f} \phi_{i}+\mathbf{L}_{\mathbf{g}} \phi_{i} \mathbf{u} \leq 0\right\}: \mathbf{x} \in \partial \Phi_{i} \wedge \mathbf{L}_{\mathbf{g}} \phi_{i} \neq \mathbf{0}_{m^{\prime}}^{\top} \\
\text { empty }: \mathbf{x} \in \partial \Phi_{i} \wedge \mathbf{L}_{\mathbf{g}} \phi_{i}=\mathbf{0}_{m^{\prime}}^{\top} \wedge L_{f} \phi_{i}>0 \\
\mathbf{u}=\text { free }: \mathbf{x} \in \partial \Phi_{i} \wedge \mathbf{L}_{\mathbf{g}} \phi_{i}=\mathbf{0}_{m^{\prime}}^{\top} \wedge L_{f} \phi_{i} \leq 0 \\
\mathbf{u}=\text { free }: \mathbf{x} \in \Phi \backslash \partial \Phi,
\end{array}\right.
\end{aligned}
$$




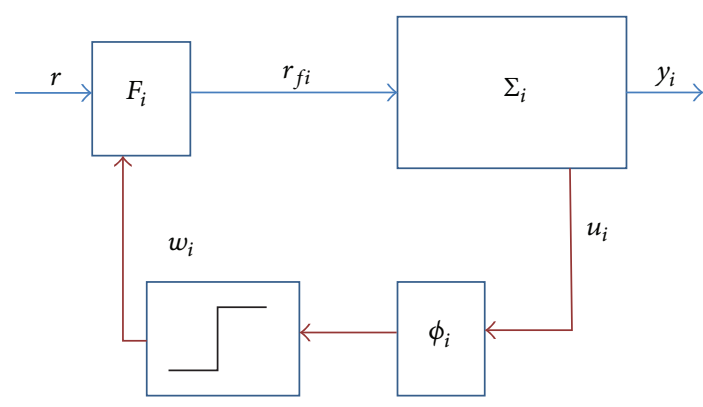

FIGURE 2: SM reference conditioning general scheme.

where $\mathbf{0}_{m}^{\top}$ denotes the $m$-dimensional null column vector, and the first set corresponding to $\mathbf{L}_{\mathbf{g}} \phi_{i} \neq \mathbf{0}_{m}^{\top}$ is always nonempty.

Note that the control $\mathbf{u}$ in the interior of $\Phi$ can be freely assigned. Particularly, $\mathbf{u}=\mathbf{0}_{m}^{\top}$ could be taken so that the system evolves autonomously throughout the interior of $\Phi$. Then, the control action becomes active only when some constraint becomes active, that is, when the state trajectory reaches the boundary $\partial \Phi$ trying to leave the set $\Phi$. The invariance condition will hold if the intersection $\bigcap_{i} \mathscr{U}_{i}(\mathbf{x})$ for all constraints of the solution sets $\mathscr{U}_{i}(\mathbf{x})$ is not empty.

3.2. Sliding Mode Reference Conditioning. Now, in order to find the necessary $\mathbf{u}$ to achieve the invariance of some set $\Phi(y)$ (3), with the system $\Sigma(2)$, consider the following implementation (Figure 2).

A discontinuous decision block will drive the search to find $\mathbf{u} \in \mathscr{U}_{i}(\mathbf{x})$ so as to fulfill the constraint $\Phi(y)$ and make $\mathbf{r}_{f}$ remain as close as possible to the external signal $\mathbf{r}$. Also, a filter $F$ is incorporated. Its purpose is to filter out the conditioned signal $\mathbf{r}_{f}$ in order to feed the system $\Sigma$ with a smooth signal.

The filter $F$ is implemented as the first-order filter

$$
\dot{\mathbf{r}}_{f}=-\Lambda\left(\mathbf{r}_{f}+\mathbf{u}-\mathbf{r}\right),
$$

where $\mathbf{u} \in \mathbf{U} \subset \mathbb{R}^{m}$ is the discontinuous control action, $\mathbf{r}, \mathbf{r}_{f} \in$ $\mathbb{R}^{m}$ are the input and the conditioned input signals, and $\Lambda \epsilon$ $\mathbb{R}^{m \times m}$ is a diagonal matrix, a design parameter of the filter.

The discontinuous decision block is implemented by means of the variable structure control law:

$$
u= \begin{cases}0 & \text { if } \max _{i}\left\{\phi_{i}(\mathbf{y})\right\} \leq 0 \\ \mathbf{u}_{\mathrm{SM}} & \text { otherwise }\end{cases}
$$

where $\phi_{i}(\mathbf{y})$ are the constraints defined previously, that is, the boundaries of the set $\Phi$, and $\mathbf{u}_{\mathrm{SM}}$ is such that $u \in \bigcap_{i} \mathscr{U}_{i}(\mathbf{x})$.

Notice that the block $\Sigma$ in Figure 2 represents the entire dynamics from the constrained variables $(\mathbf{y})$ to the input signal $\mathbf{u}$. Then system (2) becomes

$$
\begin{gathered}
\dot{\mathbf{x}}=\mathbf{f}(\mathbf{x}, \mathbf{d})+\mathbf{g}(\mathbf{x}) \mathbf{r}_{f}, \\
\dot{\mathbf{r}}_{f}=-\Lambda\left(\mathbf{r}_{f}+\mathbf{u}-\mathbf{r}\right), \\
\mathbf{y}=\mathbf{h}(\mathbf{x}) .
\end{gathered}
$$

In the case of a control system, (2) is the plant dynamic together with a control loop, in which case $\mathbf{r}_{f}$ is the reference, and $\mathbf{x}$ in (2) is the extended state comprising the plant and controller.

The choice of $\mathbf{u}_{\mathrm{SM}}$ depends on whether there is only one active constraint or more than one. For a single active constraint, the analysis is very similar to that of an SMRC in an SISO system $[17,24,25]$ and is the approach we will use hereafter. The case of several active constraints (see [22]) is not analyzed in this work since we are defining only one constraint per system in the formation control problem.

\section{Reference Coordination under Local Topology}

In this section, the local topology scheme for systems coordination is presented. The coordination problem approached is the second one described in Section 2. To this end, Definition 3 is rewritten in the following way.

Definition 5. The local objective between two connected systems is to bound the distance between their references by a predefined value $\delta_{i j}$. The set $\Phi_{i j}$ is defined by.

$$
\begin{array}{r}
\Phi_{i j}\left(\mathbf{x}, r_{f k}\right)=\left\{\mathbf{x} \in \mathbf{X}, r_{f k} \in \mathbb{R}, k=1,2:\right. \\
\left.\phi_{i j}=\left|r_{f i}-r_{f j}\right|-\delta_{i j} \leq 0\right\},
\end{array}
$$

where $\mathbf{x} \in \mathbf{X} \in \mathbb{R}^{n}$ are the systems states and $r_{f i} \in \mathbb{R}, r_{f j} \in \mathbb{R}$ are the conditioned references of systems $i$ and $j$, respectively.

Moreover, the systems are connected by the following assumption.

Assumption 6. The topology of the connection network is fixed, in the sense of which system can communicate with each other. This network can be represented by a directed graph with adjacency matrix $A=\left[a_{i j}\right]$, with $a_{i j}=1$, when $i$ th system can communicate with $j$ th one. Otherwise $a_{i j}=0$. This graph is assumed to be connected [4].

Then, the set $\Phi$ is defined as the union of the $\Phi_{i j}$, when they are connected $\left(a_{i j}=1\right)$, for all the systems in the group:

$$
\Phi=\bigcup_{i=1, j=1, i \neq j}^{N} a_{i j} \Phi_{i j} .
$$

The coordination objective is to make the set $\Phi$ a controlled invariant set. To this end, the following local coordination scheme is proposed.

4.1. Proposed Coordination Scheme. Consider a swarm of $N$ systems, with $N \geq 2$ satisfying Assumption 2, connected in a network topology as in Assumption 6, with a sliding mode reference conditioning auxiliary loop, like in Section 3.2. This loop allows us to handle the local constraints of each system by commanding a feasible reference to each system closed loop. Also, the feasible reference provides information to the other systems. The systems will have a goal for every connected neighbor systems as in Definition 5. Then, it is 
possible to enforce coordination as in Section 2 among the system references using the following scheme incorporating the local goal into each system SMRC loop.

In Figure 3, an implementation of the proposed scheme is depicted, with two systems for the sake of demonstration. The $i$ th system $\Sigma_{i}$ incorporates the plant and a biproper controller:

$$
\Sigma_{i}:\left\{\begin{array}{l}
\dot{x}_{i}=f_{i}\left(x_{i}\right)+g_{i}\left(x_{i}\right) v_{i} \\
y_{i}=h_{i}\left(x_{i}\right) \\
\dot{x}_{c i}=A_{c i} x_{c i}+b_{c i} e_{i} \\
v_{i}=c_{c i} x_{c i}+d_{c i} e_{i},
\end{array}\right.
$$

where $x_{c i}$ and $e_{i}$ are the state of the controller and the error signal of the control loop defined as $e_{i}=r_{f i}-y_{i} . A_{c i}, b_{c i}, c_{c i}$, and $d_{c i}$ are the parameters of the $i$ th system controller.

The local goal, incorporated in the SMRC, together with the actuator saturation constraint, leads to the following structure for the filter $F_{i}$ :

$$
F_{i}: \dot{r}_{f i}=-\alpha_{i}\left(r_{f i}-r+\widetilde{w}_{i}\right)
$$

with the combination function $\widetilde{w}_{i}$. This will combine the discontinuous actions from the physical constraints $\left(w_{i}\right)$ and from the virtual coordination constraints $\left(w_{i j}\right)$, defined as follows:

$$
\widetilde{w}_{i}=w_{i}+\sum_{j=1, j \neq i}^{N} a_{i j} w_{i j}
$$

The discontinuous action $w_{i}$ is defined as in [18]:

$$
w_{i}= \begin{cases}M_{i} & \text { if } \phi_{i}^{+}=v_{i}-v_{i p}^{+}>0 \\ -M_{i} & \text { if } \phi_{i}^{-}=v_{i}-v_{i p}^{-}<0 \\ 0 & \text { otherwise, }\end{cases}
$$

with $M_{i}$ being the amplitude of the discontinuous action. And the discontinuous action $w_{i j}$ is defined according to the virtual constraint $\phi_{i j}$ :

$$
w_{i j}= \begin{cases}M_{i j} \operatorname{sign}\left(r_{f i}-r_{f j}\right) & \text { if } \phi_{i j}\left(r_{f i}, r_{f j}\right) \geq 0 \\ 0 & \text { if } \phi_{i j}\left(r_{f i}, r_{f j}\right)<0,\end{cases}
$$

with $M_{i j}$ being the amplitude of the discontinuous action. Finally the physical and vitrual coordination constraints are:

$$
\begin{gathered}
\phi_{i}^{ \pm}=v_{i}-v_{i p}^{ \pm} \\
\phi_{i j}=\left|r_{f i}-r_{f j}\right|-\delta_{i j},
\end{gathered}
$$

with $v_{i p}^{+}$and $v_{i p}^{-}$being the upper and lower limits of the actuator saturation and $\delta_{i j}$ being a preestablished value for the references difference, and (19) being defined according to (11).

In Appendix B, the corresponding analysis is done to prove the invariance of the set $\Phi_{i j}$, obtaining the following bound:

$$
\begin{gathered}
M_{i j}>-\frac{\alpha_{i} r_{f i}-\alpha_{j} r_{f j}}{\alpha_{i}+\alpha_{j}}-\frac{\alpha_{i}-\alpha_{j}}{\alpha_{i}+\alpha_{j}} r, \\
M_{i}>\sum_{i \neq j} M_{i j}-\frac{\bar{b}_{c i} v_{i p}^{ \pm}+\bar{A}_{c i} x_{c i}}{b_{i}}-\rho_{i} .
\end{gathered}
$$

\section{Reference Coordination under Global Topology}

To address the coordination problem under the global topology, Definition 3 is rewritten as follows.

Definition 7. The coordination objective can be defined in terms of a set $\Phi_{\chi}$, which by changing $r$ becomes an invariant set. The set $\Phi_{\chi}$ is defined by

$$
\begin{aligned}
\Phi_{\chi}\left(\mathbf{x}, r_{f i}\right)=\left\{\mathbf{x} \in \mathbf{X}, r_{f i}\right. & \in \mathbb{R}^{N}: \\
& \left.\phi_{\chi}\left(r_{f i}\right)=\left|r-\chi\left(r_{f i}\right)\right|-\Delta \leq 0\right\},
\end{aligned}
$$

where $\mathbf{x} \in \mathbf{X} \in \mathbb{R}^{n}$ are the systems states, $r \in \mathbb{R}$ is the global conditioned references, and $\chi\left(r_{f i}\right)$ is a function of each system's reference. Finally, $\Delta$ is a fix predetermined value, the width of the allowed band around $r$.

Thus, the coordination objective is to make $\Phi_{\chi}$ become an invariant set. To this end, the following coordination scheme is proposed.

5.1. Proposed Coordination Scheme. Consider a set of $N$ dynamical systems. Assumption 2 holds for each system. Defining the coordination objective as in Definition 7, it follows that coordination understood as in Section 2 is enforced using the following scheme and incorporating the coordination objective (Definition 7) in a global reference conditioning loop.

The proposed coordination scheme is shown in Figure 4. Each agent $i$ has a local reference conditioning loop $\left(\phi_{i}, w_{i}\right.$, and $F_{i}$ like in Figure 2) to generate the feasible reference $r_{f i}$ from the global reference $r$.

In a higher hierarchical level, there is another reference conditioning loop, in this case to generate the global reference $r$. This loop comprises the switching function $\phi_{\chi}$, the function $\chi$ of the local references, and the discontinuous action $\left(w_{g}\right)$ to create a smooth global reference $r$ from the global target signal $c_{g}$.

5.1.1. Coordination Filter. The coordination filter $\left(F_{g}\right)$ smooths the global discontinuous action $\left(w_{g}\right)$ with the following dynamics:

$$
\dot{r}=-\lambda\left(r-c_{g}-k w_{g}\right)
$$

where $w_{g}$ is the discontinuous action and $k$ is a weight to define the strength of the coordination.

5.1.2. Definition of the Switching Surface $\phi_{\chi}$. First we define the boundaries of the set $\Phi_{\chi}$ as constraints in the following way:

$$
\begin{aligned}
& \phi_{\chi}^{+}=r-\chi\left(r_{f i}\right)-\Delta, \\
& \phi_{\chi}^{-}=r-\chi\left(r_{f i}\right)+\Delta .
\end{aligned}
$$




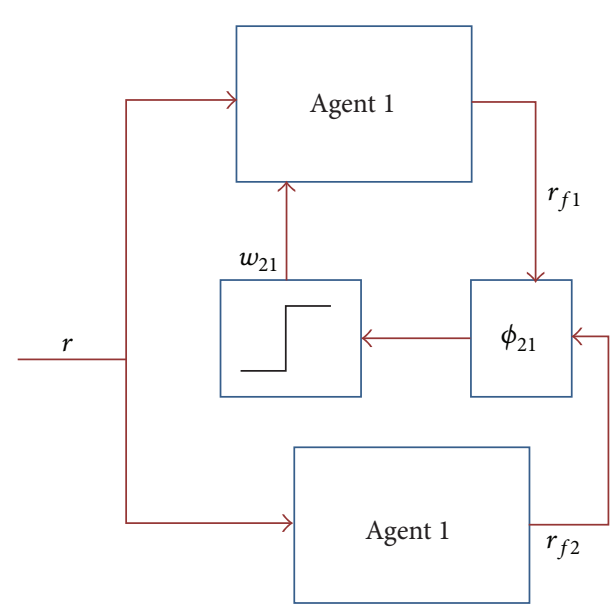

FIGURE 3: Local SMRCoord: local goal incorporation into the SMRC scheme.

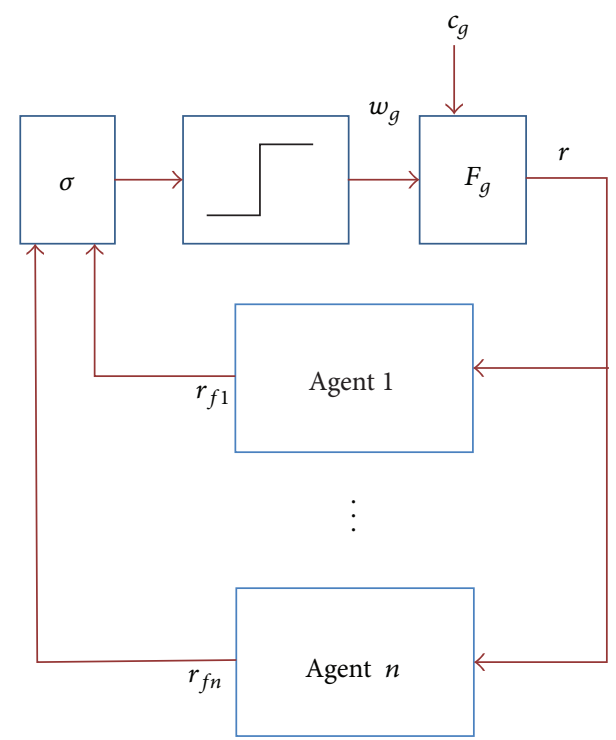

FIGURE 4: Coordination scheme for the global topology.

The discontinuous action to force the system remain in the set $\Phi_{\chi}$ when the system dynamics make the trajectories go outside of the set is

$$
w_{g}= \begin{cases}w_{g}^{+} & \text {if } \phi_{\chi}^{+}>0 \\ w_{g}^{-} & \text {if } \phi_{\bar{\chi}}^{-}<0 \\ 0 & \text { otherwise }\end{cases}
$$

To ensure the invariance of the set $\Phi_{\chi}$ (7), we must choose $w_{g}^{-} \leq-w_{g}^{\star}$ and $w_{g}^{+} \geq w_{g}^{\star}$, where $w_{g}^{\star}$ is the bound obtained in Appendix A.

\section{Simulation}

In this section, the main features of the sliding mode reference coordination (SMRCoord) and formation control developed in Sections 5 and 4 are illustrated for quadrotors with cartesian control through simulation results obtained using MATLAB and using non-linear identified models from [31]. A simplified version of the Parrot AR-Drone quadrotor models and software for development and implementation of control and navigation strategies can be found in [32]. Note the strategy is straightforward to implement in MIMO systems, as soon as they are decoupled. This is the case of the quadrotors in cartesian configuration.

6.1. Quadrotors and Controllers. In this example we considered three quadrotors. The kind of motion considered is cartesian (planar motion). Each one has its own local reference conditioning scheme as in Section 3.2. Each agent $i$ sends and receives information from the other agents under the local topology and to the supervisor under the global topology and incorporates them as constraints (Definition 5) in the SMRC as proposed in Section 4.

6.2. Information Exchange in SMRCoord. The way the information exchange is considered in this work is a key contribution. The main idea is that each system sends key information regarding its local constraints to the other systems by sending its feasible reference. In any of the two topologies, the individual systems hide their states and output to only share the feasible reference with the other systems and thus minimizing the information exchange. The feasible reference contains information of the system and its state regarding the local constraints.

\subsection{Simulation Results}

6.3.1. UAV Formation with SMRCoord under Local Topology. First we implement a triangle-shape formation with three drones using the SMRCoord under a local topology. The time evolution of the three agents references $\left(r_{f i}\right)$ together with the resulting discontinuous actions $(w)$ is shown in Figure 5.

It can be seen that the references are coordinated and follow the target trajectory taking into account the saturation in the drones actuators.

In the resulting discontinuous action (plots 3 to 5 in Figure 5), it is possible to see that the amplitude in each component is varying with time, as the constraints $\phi$ also change, depending on the relative positions of the agent between them. Also the amplitude of the discontinuous action is enough to make (21) hold, so that the SM is established in the boundary $\partial \phi$.

In Figure 6, it is possible to see how, despite the dynamical difference among the systems and their local controllers parameters, coordination is also achieved in the output of the systems.

6.3.2. Formation Perturbation Rejection with SMRCoord under Local Topology. Simulation results in order to test the formation perturbation rejection properties of the SMRCoorc under local topology can be seen in Figure 7. The perturbation consisted in manually stopping the movement of Drone 1 (red line) in the coordinate $x$ and has been incorporated after $20 \mathrm{sec}$ and after $50 \mathrm{sec}$. It appears as a flattop in the reference (surrounded by a black ellipse in the plot). The strategy rejects the perturbation as the other two drones wait; that is, they stop moving as well in order to keep 


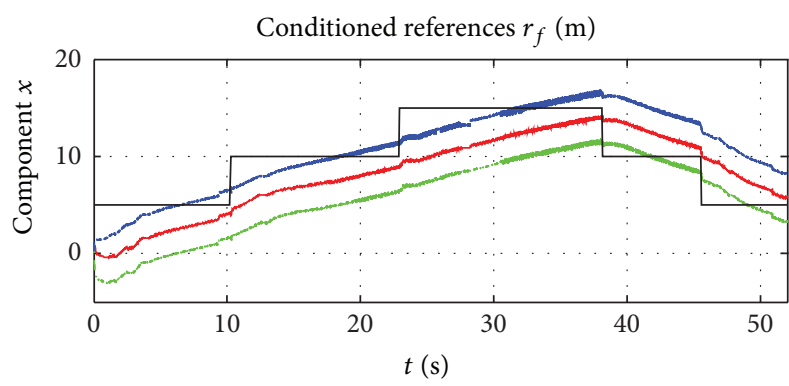

(a)

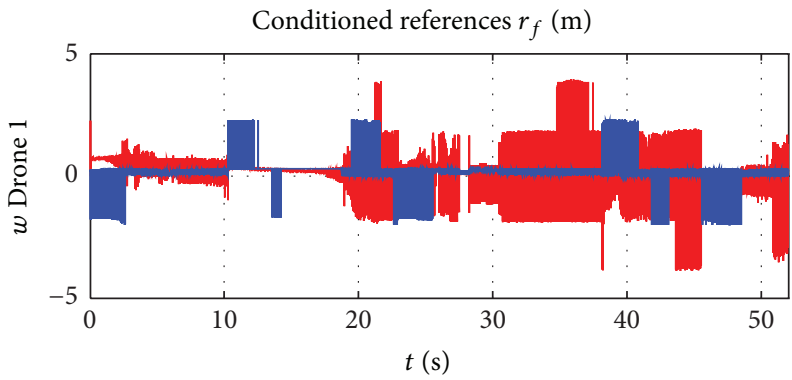

(c)

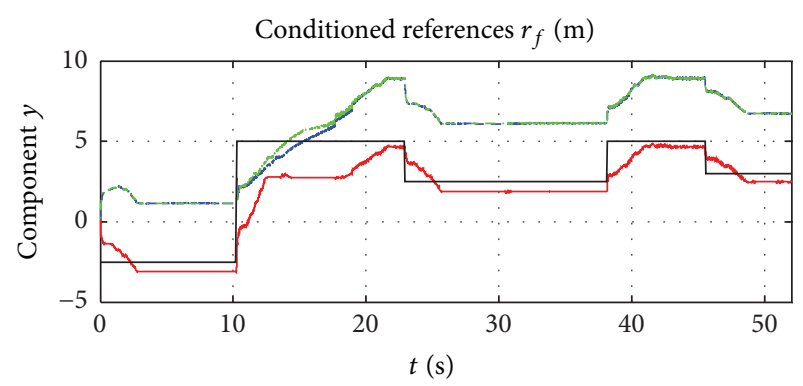

(b)

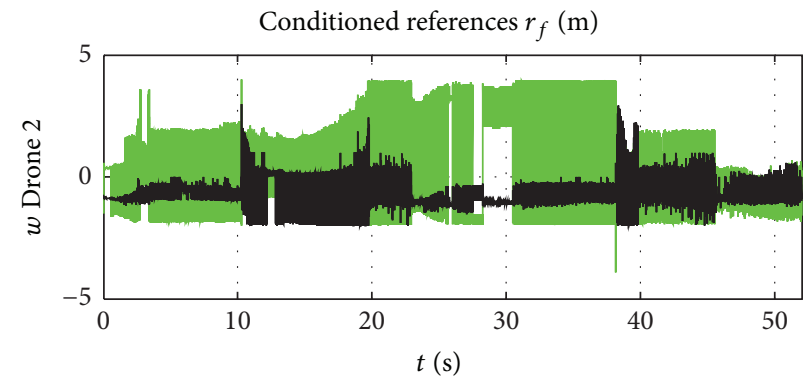

(d)

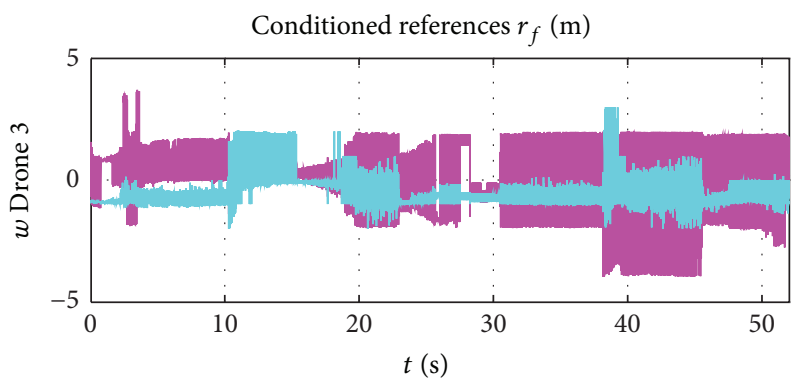

(e)

FIGURE 5: Local topology: conditioned references and discontinuous actions of the individual systems.

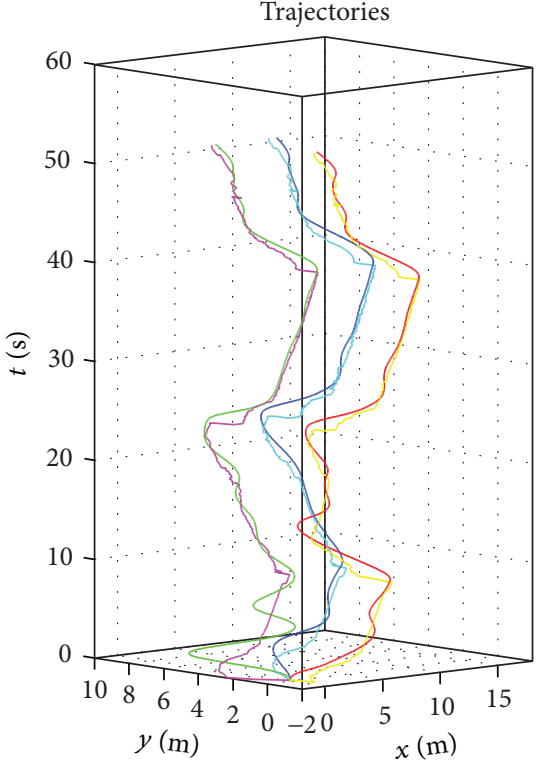

(a)

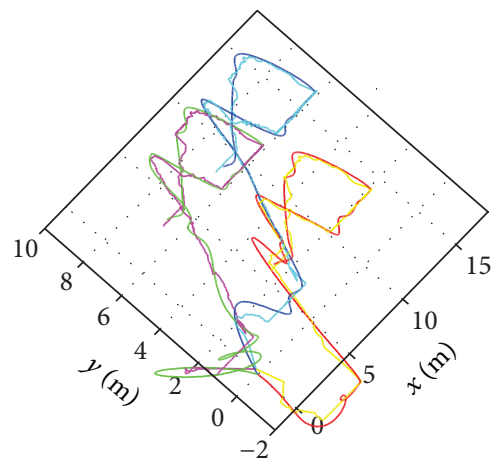

- Drone 1

Drone 2

(b)

FIGURE 6: Local topology: drones trajectories in time and cartesian projection. The trajectories are winding along the conditioned references. 


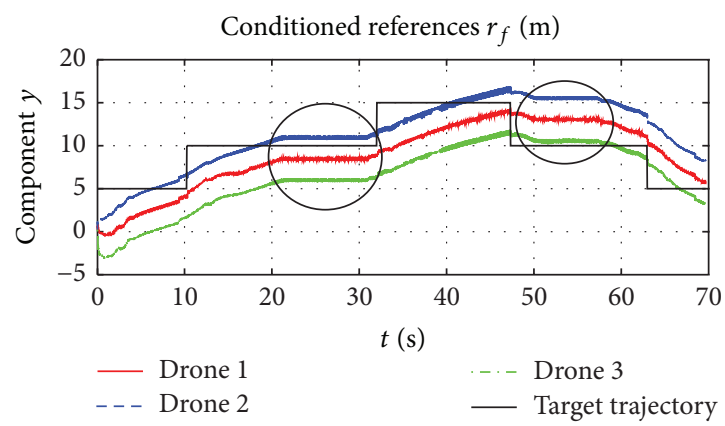

(a)

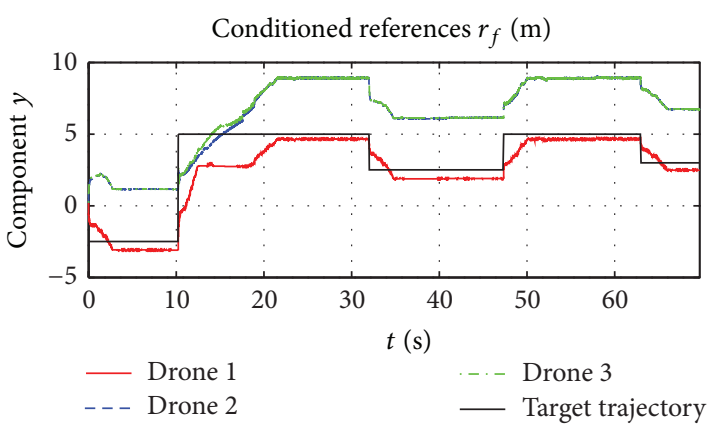

(b)

FIGURE 7: Target and conditioned references for $x$ and $y$ components under perturbations in the formation. The black ellipses show the intervals where the perturbation was introduced to Drone 1 (red), and it is possible to see how the other two conditioned references wait for the reference of Drone 1 until the perturbation vanishes, and then the normal course continues.

the formation. As a drawback, comparing with the first plot of Figure 5, the time it takes to finish the desired trajectory is longer because the drones had to wait until the perturbation was gone.

\section{Conclusion}

A novel strategy using ideas of set invariance and sliding mode reference conditioning is developed to deal with the coordination of multi-agents and formation control problem. The proposed methodology has an interesting potential to be expanded in order to overcome more general coordination problems. This is inherent to its definition; that is, the coordination goals are reflected in the design of the sliding manifolds.

The fact that the individual systems dynamics are hidden to the coordination system, while only the necessary information about the subsystems constraints is communicated to it, makes the proposed methodology transparent and allows dealing with a broad kind of systems to be coordinated, as soon as they can be reference conditioned.

Additionally, the features of the SMRC and the SM itself are inherited by the proposal, such as robustness properties of SM control but not the usual problems of SM like chattering, because the technique is implemented as a part of a numeric algorithm in a digital environment.

Practical applications of the proposed algorithm, for example, in AR-Drone quadrotors flying in a controlled formation, can be implemented as auxiliary supervisory loops to the trajectory planning algorithm for the virtual leader and stabilizing controllers of the individual agents. The research group is working towards this implementation.

In the theoretical side, an interesting future research line on which we are working is on the extensions of the proposed methodology to deal with multiple constraints per system, in which case the problem is how to decide the direction of the control action. Also we are working on the case of constrained systems and how to incorporate these constraints into the coordination in order to have a formation control which also takes care of the individual systems constraint, resulting in a robust formation control against disturbances coming from individual limitations in the multi-agent systems.

\section{Appendices}

\section{A. Global Topology Analysis}

Consider the case of having $N=2$, two individual systems, for the sake of clarity. The state space representation of the closed-loop system (when the reference conditioning loop is active) is

$$
\dot{\mathbf{x}}=f(x)+g(x) w_{g}+h(x)+\mathbf{p}
$$

with

$$
\begin{gathered}
x=\left[\begin{array}{c}
r_{f 1} \\
r_{f 2} \\
r
\end{array}\right], \quad f(x)=\left[\begin{array}{c}
-\alpha_{1}\left(r_{f 1}-r\right) \\
-\alpha_{2}\left(r_{f 2}-r\right) \\
-\lambda r
\end{array}\right], \\
g(x)=\left[\begin{array}{c}
0 \\
0 \\
-\lambda
\end{array}\right], \quad h(x)=\left[\begin{array}{c}
-\alpha_{1} w_{1} \\
-\alpha_{2} w_{2} \\
0
\end{array}\right], \quad \mathbf{p}=\left[\begin{array}{c}
0 \\
0 \\
\lambda c_{g}
\end{array}\right],
\end{gathered}
$$

where $f$ is the drift vector field, $g$ is the control vector field, $\mathbf{p}$ acts as a perturbation, and $h$ comprises the discontinuous actions of the internal conditioning loops, which are not considered as perturbations because these two signals are responsible for the difference between $r$ and $r_{f i}$. Calculating the gradient of the constraint $\phi_{\chi}$, we get

$$
\frac{\partial \phi_{\chi}}{\partial x}=\left[\begin{array}{lll}
-\frac{\partial \chi}{\partial r_{f 1}} & -\frac{\partial \chi}{\partial r_{f 2}} & 1
\end{array}\right]
$$

Note the difference between $\phi_{\chi}^{+}$and $\phi_{\chi}^{-}$is not reflected in their gradients; thus $\partial \phi_{\chi} / \partial x$ will be used to refer to either $\phi_{\chi}^{+}$'s gradient or $\phi_{\chi}^{-}$'s gradient.

The Lie derivative of $\phi_{\chi}$ in the direction of $f$ is

$$
L_{f} \phi_{\chi}=\frac{\partial \chi}{\partial r_{f 1}} \alpha_{1}\left(r_{f 1}-r\right)+\frac{\partial \chi}{\partial r_{f 2}} \alpha_{2}\left(r_{f 2}-r\right)-\lambda r
$$


and in the direction of $g$ is

$$
L_{g} \phi_{\chi}=-\lambda .
$$

Then to ensure the invariance of the set $\Phi_{\chi}$, the explicit invariance condition (7) must hold. When the filter $F_{g}$ is stable, then $\lambda>0$ and

$$
L_{g} \phi_{\chi}=-\lambda<0,
$$

which ensures (7) with

$$
\begin{aligned}
w_{g}^{\phi_{\chi}}= & \frac{\alpha_{1}}{\lambda} \frac{\partial \chi}{\partial r_{f 1}} r_{f 1}+\frac{\alpha_{2}}{\lambda} \frac{\partial \chi}{\partial r_{f 2}} r_{f 2} \\
& -\left(\frac{\alpha_{1}}{\lambda} \frac{\partial \chi}{\partial r_{f 1}}+\frac{\alpha_{2}}{\lambda} \frac{\partial \chi}{\partial r_{f 2}}+1\right) r .
\end{aligned}
$$

Consider a fixed constant $\left|w_{g}^{+}\right|,\left|w_{g}^{-}\right|<\infty$, and $w_{g}^{\star}>$ 0 . If $F_{g}$ is a filter BIBO stable with bounded input $\left|w_{g}\right| \leq$ $\max \left\{\left|w_{g}^{+}\right|,\left|w_{g}^{-}\right|\right\}=w_{g}^{\star}$ and $c_{g}$ (assuming a bounded global target), note that $|r|<K_{r}$. Since the filters $F_{i}$ also have bounded inputs $\left(r\right.$ and $\left.w_{i}\right)$, then $\left|r_{f i}\right|<K_{r_{f i}}$.

Also the bound on $\left\|\partial \chi / \partial r_{f i}\right\|<K_{\chi_{i}}$ is necessary but only depends on the selection of function $\chi$.

Then, $\exists w_{g}^{\sigma_{\chi}}$ such as

$$
\left|w_{g}^{\phi_{x}}\right| \leq \frac{1}{\lambda}\left[\sum_{i=1}^{N}\left(K_{r_{i}} K_{\chi_{i}}\right)+K_{r}\right] \leq K \leq w_{g}^{\star},
$$

and it is possible to choose $w_{g}^{-} \leq-w_{g}^{\star}$ and $w_{g}^{+} \geq w_{g}^{\star}$ from the previous inequality to ensure the invariance of the set $\Phi_{\chi}$.

\section{B. Local Topology Analysis}

The extended dynamics of the system will be analyzed in order to design the SMRC parameters of the local topology. Assuming the systems start from inside the invariant set, that is, all constraints are met, their trajectories evolve up so they reach the boundary; then the corresponding constraint is activated forcing the system to slide on the boundary surface until the system by itself comes back inside the invariant set. This analysis is done with the $i$ th systems, considering information coming from the $j$ th one.

For the analysis, let us first rewrite the $i$ th system controller and filter dynamics as follows. From (13) and (14) and using $e_{i}=d_{c i}^{-1}\left(v_{i}-c_{c i} x_{c i}\right)$, it follows that

$$
\begin{aligned}
& \dot{x}_{c i}=\left(A_{c i}-b_{c i} d_{c i}^{-1} c_{c i}\right) x_{c i}+b_{c i} d_{c i}^{-1} v_{i} \\
\dot{v}_{i}= & c_{c i}\left(A_{c i}-b_{c i} d_{c i}^{-1} c_{c i}-\alpha_{i}\right) x_{c i} \\
+ & \left(c_{c i} b_{c i} d_{c i}^{-1}-\alpha_{i}\right) v_{i}-d_{c i} \alpha_{i} \widetilde{w}_{i}+d_{c i} \alpha_{i}\left(r+y_{i}\right) \\
& -d_{c i} \dot{y}_{i} .
\end{aligned}
$$

Therefore, consider the extended state

$$
x_{i e} \triangleq\left[x_{i}, x_{c i}, v_{i}\right]^{T} \in \mathbb{R}^{n+n_{c}+1} .
$$

The joint dynamics are given in

$$
\dot{x}_{i e}=\left[\begin{array}{c}
f_{i}\left(x_{i}\right)+g_{i}\left(x_{i}\right) v_{i} \\
\left(c_{c i} \bar{b}_{c i}+\alpha_{i}\right) v_{i}+c_{c i}\left(\bar{A}_{c i}-\alpha_{i}\right) x_{c i} \\
\bar{b}_{c i} v_{i}+\bar{A}_{c i} x_{c i}+b_{i} \rho_{i}
\end{array}\right]+\left[\begin{array}{l}
0 \\
0 \\
b_{i}
\end{array}\right] \widetilde{w}_{i},
$$

with

$$
\begin{gathered}
\bar{A}_{c i}=\left(A_{c i}-b_{c i} d_{c i}^{-1} c_{c i}\right) \\
\bar{b}_{c i}=b_{c i} d_{c i}^{-1} \\
b_{i}=-d_{c i} \alpha \\
\rho_{i}=b_{i}^{-1}\left[d_{c i} \alpha_{i}\left(r-y_{i}\right)-d_{c i} \dot{y}_{i}\right] .
\end{gathered}
$$

To make the invariance condition (7) hold for constraint $\phi_{i}^{+}$, we have from (18)

$$
\begin{gathered}
\frac{\partial \phi_{i}^{ \pm}}{\partial x_{i e}}=\left[\begin{array}{lll}
0 & 0 & 1
\end{array}\right], \\
w_{i}^{\phi_{i}}=-\frac{\bar{b}_{c i} v_{i p}^{ \pm}+\bar{A}_{c i} x_{c i}}{b_{i}}-\rho_{i} .
\end{gathered}
$$

Now for the virtual constraints, let us first incorporate the $j$ th systems and rewrite in a more convenient way the previous joint dynamics using

$$
v_{i}=c_{c i} x_{c i}+d_{c i} e_{i}=c_{c i} x_{c i}+d_{c i} r_{f i}-d_{c i} y_{i},
$$

and considering the extended state

$$
\begin{gathered}
x_{i j e} \triangleq\left[x_{i}, x_{c i}, r_{f i}, x_{j}, x_{c j}, r_{f j}\right]^{T} \in \mathbb{R}^{n_{i}+n_{c i}+1+n_{j}+n_{c j}+1} \\
\dot{x}_{i j e}=\left[\begin{array}{c}
f_{i}\left(x_{i}\right)+g_{i}\left(x_{i}\right)\left(c_{c i} x_{c i}+d_{c i} r_{f i}\right)-g_{i}\left(x_{i}\right) d_{c i} y_{i} \\
A_{c i} x_{c i}+b_{c i} r_{f i}-b_{c i} y_{i} \\
-\alpha_{i} r_{f i}+\alpha_{i} r \\
f_{j}\left(x_{j}\right)+g_{j}\left(x_{j}\right)\left(c_{c j} x_{c j}+d_{c j} r_{f j}\right)-g_{j}\left(x_{j}\right) d_{c j} y_{j} \\
A_{c j} x_{c j}+b_{c j} r_{f j}-b_{c j} y_{j} \\
-\alpha_{j} r_{f j}+\alpha_{j} r
\end{array}\right] \\
+\left[\begin{array}{c}
0 \\
0 \\
-\alpha_{i} \\
0 \\
0 \\
\alpha_{j}
\end{array}\right] w_{i j} .
\end{gathered}
$$

Remark 8. In this case, in order to express the extended joint dynamics with respect to only one discontinuous action, the fact that $w_{i j}=-w_{j i}$ has been used. 
And to make the invariance condition (7) hold for constraint $\phi_{i j}$, we have from (19)

$$
\begin{aligned}
& \frac{\partial \phi_{i j}}{\partial x_{i j e}}=\left[\begin{array}{llllll}
0 & 0 & \operatorname{sign}\left(r_{f i}-r_{f j}\right) & 0 & 0 & -\operatorname{sign}\left(r_{f i}-r_{f j}\right)
\end{array}\right], \\
& w_{i}^{\phi_{i j}}=-\frac{\alpha_{i} r_{f i}-\alpha_{j} r_{f j}}{\alpha_{i}+\alpha_{j}}-\frac{\alpha_{i}-\alpha_{j}}{\alpha_{i}+\alpha_{j}} r .
\end{aligned}
$$

Then the discontinuous signals amplitudes $M_{i}$ and $M_{i j}$ are designed using the previous analysis.

In first place, the reason why $w_{i j}$ is selected as in (17) is because $L_{g} \phi_{i j}=\operatorname{sign}\left(r_{f i}-r_{f j}\right)\left(\alpha_{i}+\alpha_{j}\right)$ changes its sign depending on $r_{f i}-r_{f j}$. Then to meet (7), $w_{i j}$ has also to change its sign in the same way. Then, $M_{i j}$ can be selected to make (B.10) hold:

$$
M_{i j}>w_{i}^{\phi_{i j}}
$$

In second place, $M_{i}$ has to be selected according to the usual procedure [17] according to (7) but large enough to dominate the rest of the discontinuous terms $M_{i j}$, as the systems constraints are required to hold everywhere, because they are more important than the virtual coordination ones. The previous statement allows us to give the systems a feasible reference that can be followed without violating their constraints.

The worst case is when the terms $\left(r_{f i}-r_{f j}\right)$ have the same sign for all $i \neq j$ and opposite to $w_{i}$; then

$$
M_{i}>\sum_{i \neq j} M_{i j}+w_{i}^{\phi},
$$

with $w_{i}^{\phi}$ from (B.6).

\section{Conflict of Interests}

The authors declare that there is no conflict of interests regarding the publication of this paper.

\section{Acknowledgments}

Research in this area is partially supported by Argentine government (ANPCyT PICT 2011-0888 and CONICET PIP 112-2011-00361), Spanish government (FEDER-CICYT DPI2011-28112-C04-01), and Universitat Politécnica de Valencia (Grant FPI/2009-21).

\section{References}

[1] W. Ren, R. W. Beard, and E. M. Atkins, "Information consensus in multivehicle cooperative control," IEEE Control Systems Magazine, vol. 27, no. 2, pp. 71-82, 2007.

[2] Y. Cao, W. Yu, W. Ren, and G. Chen, "An overview of recent progress in the study of distributed multi-agent coordination," IEEE Transactions on Industrial Informatics, vol. 9, no. 1, pp. 427-438, 2013.
[3] G. Antonelli, "Interconnected dynamic systems: an overview on distributed control," IEEE Control Systems Magazine, vol. 33, no. 1, pp. 76-88, 2013.

[4] R. Olfati-Saber, J. A. Fax, and R. M. Murray, "Consensus and cooperation in networked multi-agent systems," Proceedings of the IEEE, vol. 95, no. 1, pp. 215-233, 2007.

[5] W. He and J. Cao, "Consensus control for high-order multiagent systems," IET Control Theory and Applications, vol. 5, no. 1, pp. 231-238, 2011.

[6] L. Liu, "Robust cooperative output regulation problem for nonlinear multi-agent systems," IET Control Theory and Applications, vol. 6, no. 13, pp. 2142-2148, 2012.

[7] J. L. Pitarch, A. Sala, and C. V. Ariño, "Closed-form estimates of the domain of attraction for nonlinear systems via fuzzypolynomial models," IEEE Transactions on Systems, Man and Cybernetics B, 2013.

[8] S. Núñez, H. D. Battista, F. Garelli, A. Vignoni, and J. Picó, "Secondorder sliding mode observer for multiple kinetic rates estimation in bioprocesses," Control Engineering Practice, vol. 21, no. 9, pp. 1259-1265, 2013.

[9] L. Wu, X. Su, and P. Shi, "Sliding mode control with bounded 12 gain performance of markovian jump singular time-delay systems," Automatica, vol. 48, no. 8, pp. 1929-1933, 2012.

[10] D. Galzi and Y. Shtessel, "UAV formations control using high order sliding modes," in Proceedings of the American Control Conference (ACC '06), pp. 4249-4254, Minneapolis, Minn, USA, June 2006.

[11] Y. Cao, W. Ren, and Z. Meng, "Decentralized finite-time sliding mode estimators and their applications in decentralized finitetime formation tracking," Systems and Control Letters, vol. 59, no. 9, pp. 522-529, 2010.

[12] J. Cortés, "Finite-time convergent gradient flows with applications to network consensus," Automatica, vol. 42, no. 11, pp. 1993-2000, 2006.

[13] S. Rao and D. Ghose, "Achieving consensus amongst selfpropelling agents by enforcing sliding modes," in Proceedings of the 11th International Workshop on Variable Structure Systems (VSS '10), pp. 404-409, June 2010.

[14] S. Rao and D. Ghose, "Sliding mode control-based algorithms for consensus in connected swarms," International Journal of Control, vol. 84, no. 9, pp. 1477-1490, 2011.

[15] P. Guo, J. Zhang, M. Lyu, and Y. Bo, "Sliding mode control for multiagent system with time-delay and uncertainties: an lmi approach," Mathematical Problems in Engineering, vol. 2013, Article ID 805492, p. 12, 2013.

[16] M. Jafarian and C. de Persis, "Exact formation control with very coarse information," in Proceedings of the IEEE American Control Conference (ACC '13), pp. 3026-3031, 2013.

[17] F. Garelli, R. J. Mantz, and H. de Battista, Advanced Control for Constrained Processes and Systems, The Institution of Engineering and Technology, 2011.

[18] F. Garelli, R. J. Mantz, and H. de Battista, "Limiting interactions in decentralized control of MIMO systems," Journal of Process Control, vol. 16, no. 5, pp. 473-483, 2006.

[19] F. Garelli, R. J. Mantz, and H. de Battista, "Sliding mode compensation to preserve dynamic decoupling of stable systems," Chemical Engineering Science, vol. 62, no. 17, pp. 4705-4716, 2007.

[20] J. Picó, F. Garelli, H. de Battista, and R. J. Mantz, "Geometric invariance and reference conditioning ideas for control of overflow metabolism," Journal of Process Control, vol. 19, no. 10, pp. 1617-1626, 2009. 
[21] A. Revert, F. Garelli, J. Pico, H. de Battista, P. Rossetti, and J. Vehi, "Safety auxiliary feedback element for the artificial pancreas in type 1 diabetes," IEEE Transactions on Biomedical Engineering, vol. 60, no. 8, pp. 2113-2122, 2013.

[22] L. Gracia, A. Sala, and F. Garelli, "A supervisory loop approach to fulfill workspace constraints in redundant robots," Robotics and Autonomous Systems, vol. 60, no. 1, pp. 1-15, 2012.

[23] L. Gracia, F. Garelli, and A. Sala, "Integrated sliding-mode algorithms in robot tracking applications," Robotics and ComputerIntegrated Manufacturing, vol. 29, no. 1, pp. 53-62, 2013.

[24] A. Vignoni, J. Picó, F. Garelli, and H. de Battista, "Dynamical systems coordination via sliding mode reference conditioning," in Proceedings of the 18th IFAC World Congress, vol. 18, pp. 11086-11091, 2011.

[25] A. Vignoni, J. Picó, F. Garelli, and H. de Battista, "Sliding mode reference conditioning for coordination in swarms of nonidentical multi-agent systems," in Proceedings of the 12th IEEE International Workshop on Variable Structure Systems, pp. 231236, 2012.

[26] A. Vignoni, F. Garelli, S. Garcìa-Nieto, and J. Picó, "Uav reference conditioning for formation control via set invariance and sliding modes," in Proceedings of the 3rd IFAC Workshop on Distributed Estimation and Control in Networked Systems, vol. 3, pp. 317-322, 2012.

[27] A. Vignoni, F. Garelli, and J. Picó, "Coordinación de sistemas con diferentes dinàmicas utilizando conceptos de invarianza geométrica y modos deslizantes," Revista Iberoamericana de Automàtica e Informàtica Industral RIAI, vol. 10, no. 4, pp. 390401, 2013.

[28] R. Hanus, M. Kinnaert, and J. L. Henrotte, "Conditioning technique, a general anti-windup and bumpless transfer method," Automatica, vol. 23, no. 6, pp. 729-739, 1987.

[29] H. Amann, Ordinary Differential Equations: An Introduction to Nonlinear Analysis, Walter de Gruyter, 1990.

[30] J. Mareczek, M. Buss, and M. Spong, "Invariance control for a class of cascade nonlinear systems," IEEE Transactions on Automatic Control, vol. 47, no. 4, pp. 636-640, 2002.

[31] X. Blasco, S. Garcìa-Nieto, and G. Reynoso-Meza, "Control autónomo del seguimiento de trayectorias de un vehìculo cuatrirrotor. simulación y evaluación de propuestas," Revista Iberoamericana de Automàtica e Informàtica Industrial RIAI, vol. 9, no. 2, pp. 194-199, 2012.

[32] S. García-Nieto, X. Blasco, J. Sanchís, J. M. Herrero, G. Reynoso-Meza, and M. Martínez-Iranzo, "Trackdrone lite," 2012, http://hdl.handle.net/10251/16427 . 


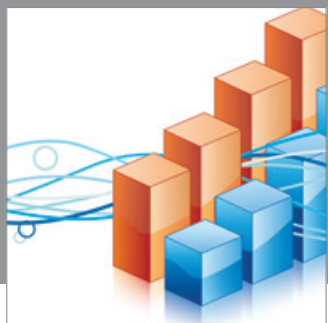

Advances in

Operations Research

mansans

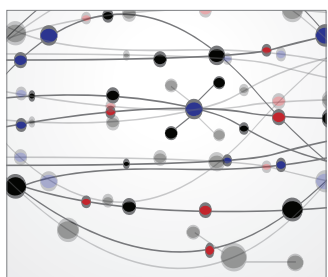

The Scientific World Journal
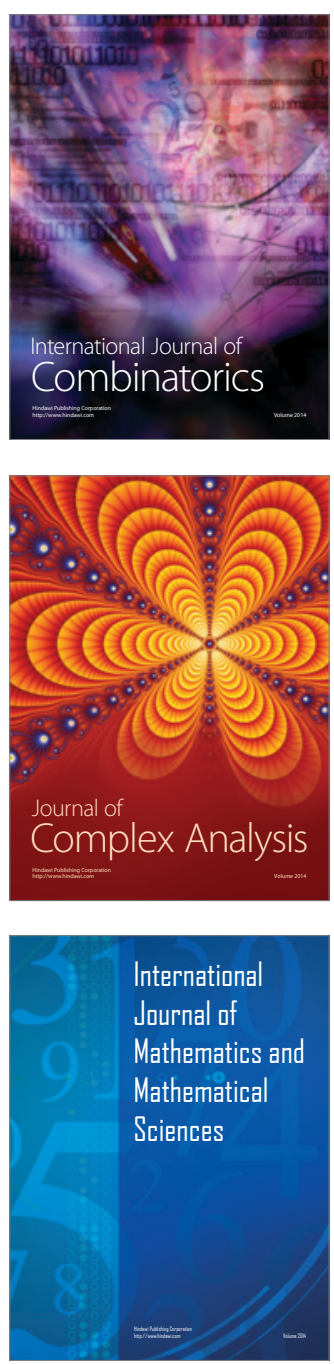
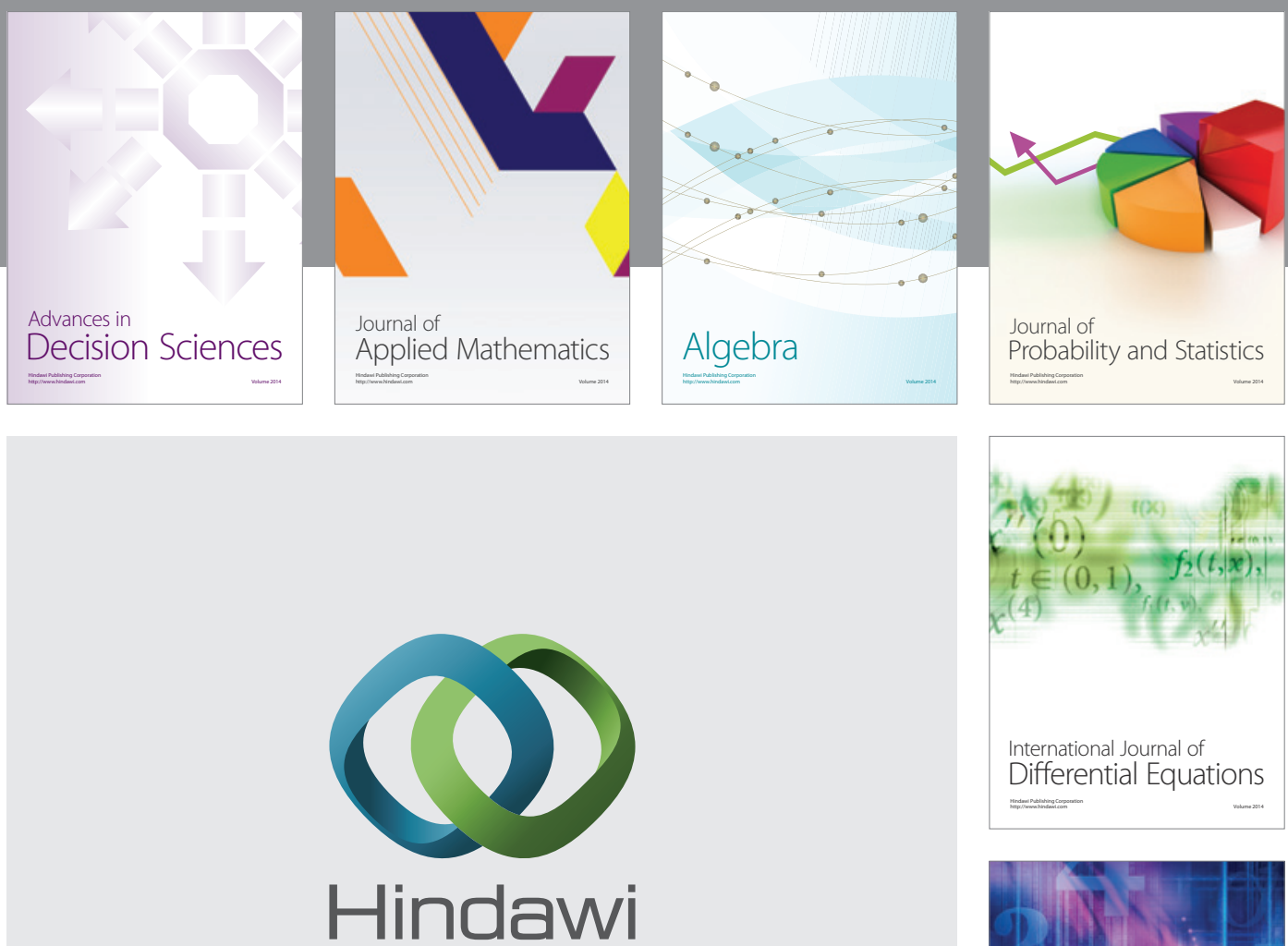

Submit your manuscripts at http://www.hindawi.com
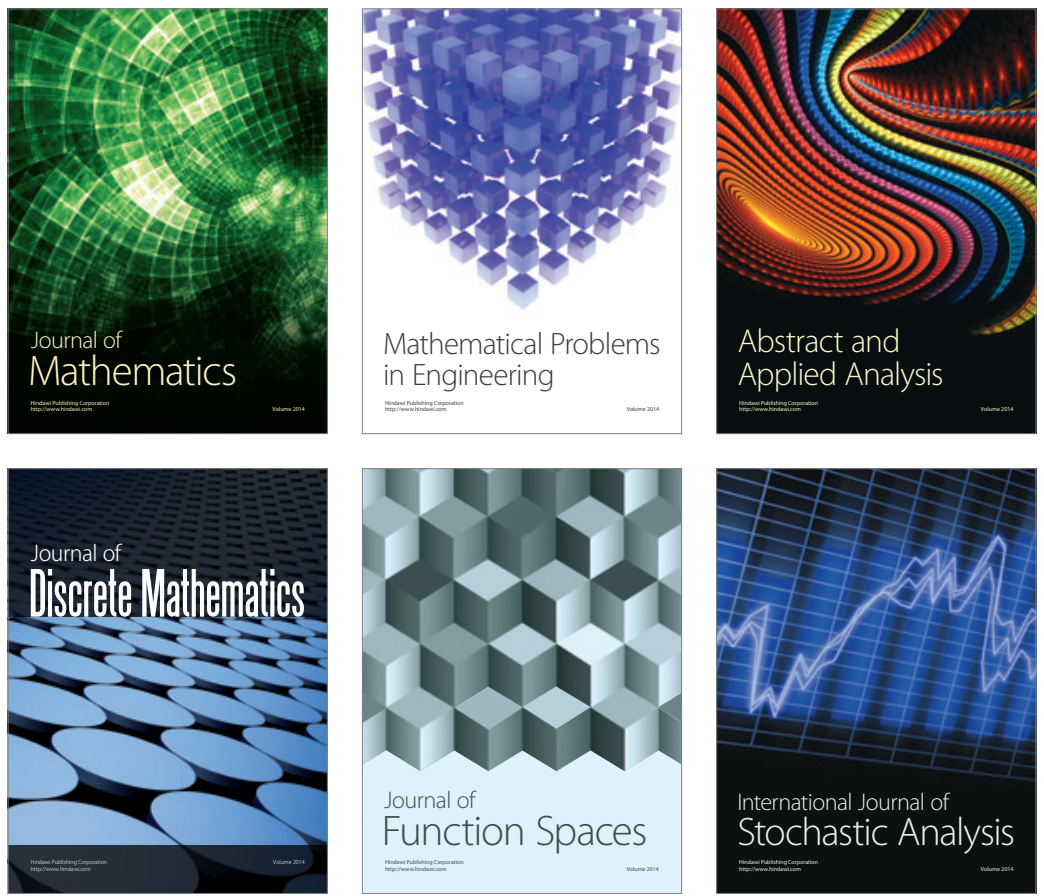

Journal of

Function Spaces

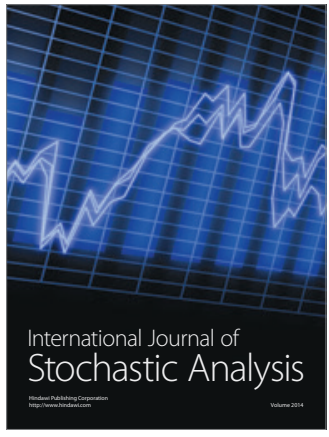

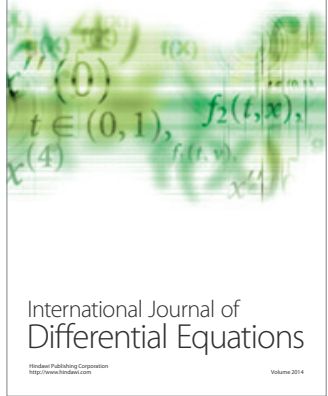
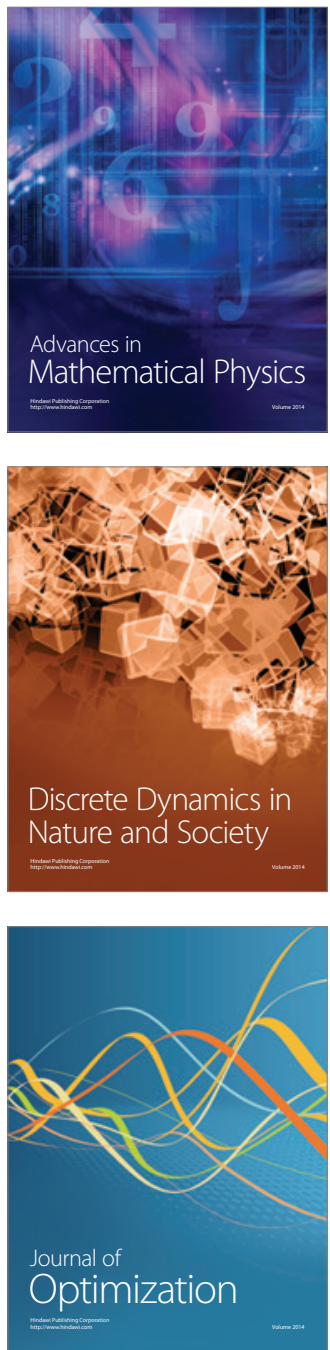